\title{
Fully Abstract Trace Semantics for Low-level Isolation Mechanisms
}

\author{
Marco Patrignani ${ }^{*} \quad$ Dave Clarke \\ iMinds-DistriNet, Dept. Computer Science, KU Leuven \\ \{first.last\}@cs.kuleuven.be
}

\begin{abstract}
Many software systems adopt isolation mechanisms of modern processors as software security building blocks. Reasoning about these building blocks means reasoning about elaborate assembly code, which can be very complex due to the loose structure of the code. A way to overcome this complexity is giving the code a more structured semantics. This paper presents one such semantics, namely a fully abstract trace semantics, for an assembly language enhanced with protection mechanisms of modern processors. The trace semantics represents the behaviour of protected assembly code with simple abstractions, unburdened by low-level details, at the maximum degree of precision. Additionally, it captures the capabilities of attackers to protected software and simplifies providing a secure compiler targeting that language.
\end{abstract}

\section{Categories and Subject Descriptors}

D.2.4 [Software/Program Verification]: Formal models

\section{INTRODUCTION}

Many processors provide isolation mechanisms as software security building blocks. These are used to withstand lowlevel attackers who, through injected assembly code, can read the whole memory space and access secrets in memory, violate integrity constraints and so on. With isolation mechanisms, attackers cannot directly violate security properties of isolated software since the isolated memory is not accessible to them. Examples of these protection mechanisms are fine grained, program counter-based memory access control mechanism $[4,8,9,12,17,18,19]$, which enforce security properties at process or lower levels (Ring-1). With this mechanism, the software to be secured is placed in a protected memory partition that shields it from the surrounding, potentially malicious code. The malicious code cannot read nor write the protected memory; it can only jump to specific addresses in protected memory to call functions of

* Marco Patrignani holds a Ph.D. fellowship from the Research Foundation Flanders (FWO).

Permission to make digital or hard copies of all or part of this work for personal or classroom use is granted without fee provided that copies are not made or distributed for profit or commercial advantage and that copies bear this notice and the full citation on the first page. To copy otherwise, to republish, to post on servers or to redistribute to lists, requires prior specific permission and/or a fee.

SAC'14 March, Gyeongju, Korea

Copyright 2014 ACM 978-1-4503-1656-9/13/03 ...\$15.00. the protected code. This protection mechanism makes software more resilient against low-level attackers. However, this does not prevent an attacker from interacting with the protected code and violating security properties.

To enable reasoning about the behaviour of isolated software, this paper firstly introduces an assembly language enhanced with a fine grained, program counter-based memory access control. This protection mechanism was chosen due to its application in secure compilation $[2,14]$ and to its recent industrial implementation: the Intel SGX [9]. Then, this paper explores the design space of trace semantics and presents two different fully abstract [15] trace semantics for the protected assembly code. The paper provides a general proof strategy where full abstraction of the trace semantics can be proven simply. To the best of the authors' knowledge, this is the first such result for an assembly language extended with the said protection mechanism. Moreover, the results seem applicable to other isolation mechanisms.

The fully abstract trace semantics provides a number of benefits. Trace semantics uses simple abstractions to represent the behaviour of protected assembly code, unburdened by low-level details, at the maximum degree of precision. So, dually, it models the behaviour of an attacker to the protected code, since it captures precisely the capabilities of that attacker. Secondly, without the trace semantics, the behaviour of protected assembly code (and thus of an attacker) is given in terms of low-level contexts. While being precise, low-level contexts are notoriously difficult to reason about, since they offer no inductive nor coinductive structure. The fully abstract trace semantics allows low-level contexts to be disregarded, since low-level contexts and traces are proven to be equally precise. Finally, the field of secure compilation benefits from the trace semantics. Recently, Agten et al. [2] and Patrignani et al. [14] presented secure compilers to assembly code enhanced with the aforementioned protection mechanism. Both results assume that the assembly language has a fully abstract trace semantics, thus the results of this paper strengthen those secure compilation claims.

In summary, the contributions of this paper are:

- the formalisation of an assembly language modelling an architecture enhanced with a fine grained, program counter-based memory access control;

- two different trace semantics for that low-level model;

- the proof that both trace semantics are fully abstract in a general proof strategy.

Limitations of this work are twofold. Firstly, the trace semantics cannot express side-channel attacks. Secondly, the 
formalisation does not consider details of the architecture such as caches; yet this is a often assumed [2, 11, 14, 16].

The paper is organised as follows. Section 2 introduces background notions for the work. Section 3 formalises syntax and operational semantics of the assembly language. Section 4 describes two trace semantics for the language. Section 5 contains the proof of full abstraction of both trace semantics. Section 6 describes related work. Section 7 discusses future works and concludes.

\section{BACKGROUND NOTIONS}

This section describes the memory access control mechanism. Then, it describes a fully abstract trace semantics for protected assembly code by turning an imprecise representation of the behaviour of the code into a precise one.

\subsection{The Protection Mechanism, Informally}

The assembly language is enhanced with an isolation mechanism: a fine-grained, program counter-based memory access control mechanism $[4,8,12,17,18,19]$. We review this mechanism from the work of Strackx and Piessens [18]. However, the techniques presented in this paper can be easily adapted to reasoning about other isolation mechanisms [4, $8,17]$. The protection mechanism provides a secure environment for running code that must be protected from the code it interacts with. This mechanism assumes that the memory is logically divided into a protected and an unprotected partition. The protected partition is further divided into a code and a data section. The code section contains a variable number of entry points: the only addresses to which instructions in unprotected memory can jump and execute. The data section is accessible only from the protected partition. For the sake of simplicity, code in protected memory cannot read the unprotected one; as discussed in Section 2.2 below. Based on the location of the program counter, instructions that violate the access control policy are ruled out. The table below summarises the access control model enforced by the protection mechanism.

\begin{tabular}{|c|c|c|c|c|}
\hline From\To & \multicolumn{3}{|c|}{ Protected } & Unprotected \\
& Entry Point & Code & Data & \\
\hline Protected & $\mathrm{r} \mathrm{x}$ & $\mathrm{r} \mathrm{x}$ & $\mathrm{r} \mathrm{w}$ & $\mathrm{w} \mathrm{x}$ \\
\hline Unprotected & $\mathrm{x}$ & & & $\mathrm{r} \mathrm{w} \mathrm{x}$ \\
\hline
\end{tabular}

\subsection{Devising Fully Abstract Trace Semantics}

Following is the notation used in all code examples throughout this paper. Every instruction in the code is preceded by the address where it is located. Call the left program $P_{L}$ and the right one $P_{R}$. When $P_{L}$ and $P_{R}$ are executed, they are placed in a protected memory partition spanning from address 100 to 200, with a single entry point at address 100 .

EXAMPLE 1.

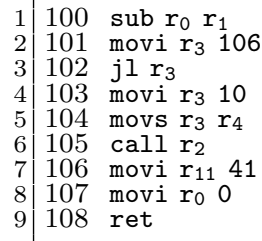

Both $P_{L}$ and $P_{R}$ assign to $r_{0}$ the result of $r_{0}-r_{1}$ (line 1 ). If the result of the operation is not less than 0 (line 3 ), they respectively write the contents of $r_{4}$ and $r_{5}$ at the unprotected address 10 (lines 4,5) and call a function whose address is stored in $r_{2}$ (line 6). Otherwise, they assign different values to $r_{11}$ (line 7) and return 0 (lines 8,9).

In order to describe the behaviour of the protected code of Example 1, this paper defines a traces semantics for it. The trace semantics gives a simple characterisation of the behaviour of that code as a set of sequences of labels. In this paper, trace semantics are devised to capture the execution of a protected program, which is a program allocated in the protected memory partition. The execution of a program is the evaluation of a sequence of instructions; in order to abstract over instructions, not all of them generate a label in a trace. Labels are generated only by call and ret instructions. If they are executed by unprotected code, they are named calls and returnbacks, if they are executed by protected code they are named callbacks and returns $[2,14]$. Following is the syntax of labels.

$$
L::=a|\tau \quad a::=g ?| g ! \quad g::=\operatorname{call} p(\bar{v}) \mid \text { ret } v
$$

A label $L$ can be an observable action $a$ or a non-observable action $\tau$. Decorations ? and ! indicate the direction of the observable action: from unprotected to protected code (?) or vice-versa (!). Address $p$ is an address in memory, $\bar{v}$ is a list of the contents of all registers and $v$ indicates the contents of register $r_{0}$.

Informally, a trace semantics is fully abstract when its labels capture all that is being communicated between the protected and the unprotected code. The trace semantics hinted at above is not fully abstract due to a number of subtleties, as highlighted by the following traces for the code of Example 1. Omitted details are indicated using ...; use . to separate actions of the same trace.

$$
\begin{aligned}
& \overline{a_{1}}=\text { call } 100(1,2, \ldots) ? \cdot \text { ret } 0 ! \\
& \overline{a_{2}}=\text { call } 100(2,1,40, \ldots) ? \cdot \operatorname{call} 40(\ldots) !
\end{aligned}
$$

Traces $\overline{a_{1}}$ and $\overline{a_{2}}$ are generated by both $P_{L}$ and $P_{R}$. Trace $\overline{a_{1}}$ does not capture the different value stored in $r_{11}$ (line 7 ), which, even if it is not the returned value of the function, still constitutes an observable difference between $P_{L}$ and $P_{R}$. Trace $\overline{a_{2}}$ does not capture the different value written at address 10 (line 5), which also constitutes a observable difference between $P_{L}$ and $P_{R}$. Since $\overline{a_{1}}$ and $\overline{a_{2}}$ do not capture the observable differences between $P_{L}$ and $P_{R}$, the trace semantics fails to be fully abstract.

As Curien stated [3], two ways to achieve full abstraction for a trace semantics exist. The first is to modify the labels so that they capture more precisely what is communicated between protected and unprotected code. In this case, labels should capture the values of all registers and what protected code writes in unprotected memory. The second is to change the operational semantics, to restrict what is communicated to what is captured by the labels. This is achieved by restricting the ways in which communication is performed, e.g. by preventing writeouts. Both approaches are presented in Section 4; they rely on an assembly language and on its operational semantics which are formalised in Section 3.

\section{LANGUAGE FORMALISATION}

This section formalises the syntax, operational semantics and contextual equivalence of an assembly language.

\subsection{Syntax}


The language is run on an architecture that models a Von Neumann machine consisting of a program counter $p$, a register file $r$, a flags register $f$ and memory space $m$. The program counter indicates the address of the instruction that is executed. The register file contains 12 general purpose registers $R_{0}$ to $R_{11}$ and a stack pointer register $S P$, which contains the address of the top of the current call stack. The flags register contains a zero flag ZF and a sign flag SF, which are set or cleared by arithmetic instructions and are used by branching instructions. For the sake of simplicity, assume the architecture targeted by the language works with $\ell$ bit-long words, where $\ell$ is a power of 2 . This allows the formalisation presented to scale to architectures with words of different sizes.

$$
\begin{array}{rrrr}
\text { Words } & w::=i \in \mathcal{I} & \text { Memories } & m::=\emptyset \\
& \mid v \in \mathcal{V} & & \mid m ; a \mapsto w \\
\text { Empty word } & \mathbf{0}::=0^{\ell} & \text { Numbers } & n::=n \in \mathbb{N} \\
\text { Addresses } & a \in 0 . .2^{\ell}-1 \quad \text { Programs } & P::=(m, s) \\
& \text { Values } & \mathcal{V}::=\left\{v \mid v=[0 \text { or } 1]^{\ell} \wedge \forall i \in \mathcal{I} . v \neq i\right\} \\
\text { Memory descriptors } & s::=\left(a_{b}, n_{c}, n_{d}, n\right) &
\end{array}
$$

\section{Figure 1: Assembly language formalisation.}

Fig. 1 presents elements of the formalisation. Words $w$ are either instructions $i$ or values $v$. Values $v$ are elements of the set $\mathcal{V}$, they are sequences of bits 0 or 1 of length $\ell$ that are different from the encoding of instructions. The explanation of why values are encoded in this way is delayed to Example 3 in Section 4.1. Instructions $i$ are elements of the set $\mathcal{I}$ and define the programming language executed on the architecture (Fig. 2). The empty word $\mathbf{0}$ is a sequence of 0 's

movl $r_{d} r_{s}$ Load the word from the memory address in register $r_{\mathrm{s}}$ into register $r_{\mathrm{d}}$.

movs $r_{d} r_{s}$ Store the contents of register $r_{s}$ at the address found in register $r_{\mathrm{d}}$.

movi $r_{d} k$ Load the constant value $k$ into register $r_{d}$. Note that $\mathrm{k}<2^{\ell}$.

add $r_{\mathrm{d}} r_{\mathrm{s}} \quad$ Write $r_{\mathrm{d}}+r_{\mathrm{s}} \bmod 2^{\ell}$ into register $r_{\mathrm{d}}$ and set the ZF flag accordingly.

sub $r_{d} r_{s}$ Write $r_{d}-r_{s} \bmod 2^{\ell}$ into register $r_{d}$ and set both the ZF and the SF flags accordingly.

cmp $r_{1} r_{2}$ Calculate $r_{1}-r_{2}$ and set both the ZF and the SF flags accordingly.

jmp $r_{i} \quad$ Jump to the address located in register $r_{i}$.

je $r_{i} \quad$ If the ZF flag is set, jump to the address in register $r_{i}$.

$j 1 r_{i} \quad$ If the SF flag is set, jump to the address in register $r_{i}$.

call $r_{i} \quad$ Push the value of the program counter +1 onto the stack and jump to the address in register $r_{i}$.

ret Pop a value from the stack and jump to the popped location.

halt Stop the execution with the result in register $r_{0}$.

Figure 2: Instruction set $\mathcal{I}$.

whose length is based on the architecture being considered. Addresses $a$ are natural numbers, ranging from 0 to $2^{\ell}-1$. Memories $m$ are maps from addresses to words. Memory access, denoted as $m(a)$, is defined as follows: $m(a)=w$ if $a \mapsto w \in m$; it is undefined otherwise. Define the domain of a memory as $\operatorname{dom}(m)=\{a \mid a \mapsto w \in m\}$. If two memories $m$ and $m^{\prime}$ have disjoint domains, they can be merged in another memory. Formally, if $\operatorname{dom}(m) \cap \operatorname{dom}\left(m^{\prime}\right)=\emptyset$, then $m+m^{\prime}=\left\{a \mapsto w \mid a \mapsto w \in m\right.$ or $\left.a \mapsto w \in m^{\prime}\right\}$. Memory descriptors $s$ are quadruples: $\left(a_{b}, n_{c}, n_{d}, n\right)$ that formalise the concepts of Section 2.1: $a_{b}$ is the address where the protected memory partition starts, $n_{c}$ and $n_{d}$ are the sizes (in number of addresses) of the code and data section respectively and $n$ is the number of entry points. Entry points are allocated starting from the base address $a_{b}$. Each entry point is $\mathcal{N}_{e}$ words long. Assume the entry points do not overflow the protected code section, thus the constraint $n \cdot \mathcal{N}_{e}<n_{c}$ holds for the all memory descriptors. Programs $P$ are pairs of a memory $m$ and a memory descriptor $s$.

\subsection{Operational Semantics}

Before introducing the semantics, a number of auxiliary notions are defined.

Fig. 3 defines the access control enforcement rules informally presented in Section 2.1. Read judgments $s \vdash$ predicate $(a, b, \cdots)$ as: "according to $s$, predicate holds for addresses $a, b, \cdots "$. Assume $s \equiv\left(a_{b}, n_{c}, n_{d}, n\right)$.

$$
\begin{aligned}
& \text { (Aux-protected) } \\
& \frac{a_{b} \leq p<\left(a_{b}+n_{c}+n_{d}\right)}{s \vdash \operatorname{protected}(p)} \\
& \text { (Aux-unprotected2) } \\
& \text { (Aux-unprotected1) } \\
& \frac{\left(a_{b}+n_{c}+n_{d}\right) \leq p}{s \vdash \operatorname{unprotected}(p)} \\
& \text { (Aux-entryPoint) } \\
& p=a_{b}+m \cdot \mathcal{N}_{e} \\
& m \in \mathbb{N} \quad m<n \\
& s \vdash \operatorname{entryPoint}(p) \\
& \text { (Aux-read-1) } \\
& s \vdash \operatorname{protected}(p) \\
& s \vdash \operatorname{protected}(a) \\
& s \vdash \operatorname{readAllowed}(p, a) \\
& \text { (Aux-entry) } \\
& s \vdash \text { unprotected }(p) \\
& s \vdash \operatorname{entryPoint}\left(p^{\prime}\right) \\
& s \vdash \operatorname{entryJump}\left(p, p^{\prime}\right) \\
& \text { (Aux-internal) } \\
& s \vdash \operatorname{protected}(p) \\
& s \vdash \operatorname{protected}\left(p^{\prime}\right) \\
& s \nvdash \operatorname{data}\left(p^{\prime}\right) \\
& s \vdash \operatorname{int} \operatorname{Jump}\left(p, p^{\prime}\right) \\
& p<a_{b} \\
& s \vdash \operatorname{unprotected}(p) \\
& \text { (Aux-returnEntry) } \\
& \frac{p=a_{b}+(n-1) \cdot \mathcal{N}_{e}}{s \vdash \operatorname{returnEntryPoint}(p)} \\
& \text { (Aux-data) } \\
& \left(a_{b}+n_{c}\right) \leq p \\
& \frac{p<\left(a_{b}+n_{c}+n_{d}\right)}{s \vdash \operatorname{data}(p)} \\
& \text { (Aux-read-2) } \\
& \begin{array}{c}
s \vdash \operatorname{unprotected}(p) \\
s \vdash \operatorname{unprotected}(a) \\
\hline s \vdash \operatorname{readAllowed}(p, a) \\
\text { (Aux-write-2) }
\end{array} \\
& s \vdash \operatorname{protected}(p) \\
& s \vdash \operatorname{data}(a) \\
& s \vdash \text { writeAllowed }(p, a) \\
& \text { (Aux-return) } \\
& s \vdash \operatorname{protected}(p) \\
& \frac{s \vdash \operatorname{unprotected}\left(p^{\prime}\right)}{s \vdash \operatorname{exitJump}\left(p, p^{\prime}\right)}
\end{aligned}
$$

Figure 3: Access control enforcement rules.

Define functions $\mathrm{m}_{\mathrm{sec}}(m, s)$ and $\mathrm{m}_{\text {ext }}(m, s)$, which return the protected and unprotected parts of a memory $m$ according to the descriptor $s$, respectively as: $\mathrm{m}_{\mathrm{sec}}(m, s)=\{a \mapsto$ $w \mid a \mapsto w \in m, s \vdash \operatorname{protected}(a)\}$ and $\operatorname{mext}_{\text {ext }}(m, s)=\{a \mapsto$ $w \mid a \mapsto w \in m, s \vdash \operatorname{unprotected}(a)\}$.

In the semantics there are two call stacks, one for the protected code, called the secure stack, and one for the unprotected code, called the insecure stack. Each stack is preceded by a word containing the location of the current head of the stack, let $\mathrm{SP}_{\mathrm{sec}}$ and $\mathrm{SP}_{\text {ext }}$ indicate the address of the secure and insecure stack respectively. When the current 
stack is changed in the semantics, the stack pointer register $\mathrm{SP}$ is initialised to the right address using $\mathrm{SP}_{\text {sec }}$ and $\mathrm{SP}_{\text {ext }}$. Given a memory descriptor $s=\left(a_{b}, n_{c}, n_{d}, n\right)$, the secure stack starts at the beginning of the protected data section and the insecure stack starts at the end of the protected memory partition. Thus $\mathrm{SP}_{\mathrm{sec}}=\left(a_{b}+n_{c}\right)$ and, initially, $\mathrm{SP}_{\mathrm{sec}} \mapsto\left(a_{b}+n_{c}+1\right)$; analogously, $\mathrm{SP}_{\mathrm{ext}}=\left(a_{b}+n_{c}+n_{d}\right)$ and, initially, $\mathrm{SP}_{\text {ext }} \mapsto\left(a_{b}+n_{c}+n_{d}+1\right)$. Call and return instructions are responsible of setting the SP register to the correct address when crossing boundaries between protected and unprotected memory. The value of the program counter is pushed onto the stack by a call instruction (the stack grows down), while a ret instruction pops one address from the top of the stack and jumps to that location. Updating the stack pointer SP is performed by the auxiliary function setStack (Fig. 4). In the rules, notation $m[a \mapsto w]$ indicates that memory $m$ is updated to a new one that is equal to $m$ except that the value stored at address $a$ is $w$. Notation $r[\mathrm{R} \mapsto w]$ indicates that the register file $r$ is updated to a new one that is equal to $r$ except that the value stored in register $\mathrm{R}$ is $w$. Notation $r(\mathrm{R})$ indicates the value contained in register $\mathrm{R}$ in register file $r$. Given a jump between

$$
\begin{aligned}
& \text { (Stack-out-to-in) } \\
& s \vdash \operatorname{entry\operatorname {Jump}}\left(p, p^{\prime}\right) \\
& m^{\prime}=m\left[\mathrm{SP}_{\text {ext }} \mapsto r(\mathrm{SP})\right] \quad r^{\prime}=r\left[\mathrm{SP} \mapsto m\left(\mathrm{SP}_{\mathrm{sec}}\right)\right] \\
& s \vdash \text { unprotected } r(\mathrm{SP}) \quad s \vdash \text { protected } r^{\prime}(\mathrm{SP}) \\
& p, r, m, s \vdash \text { setStack } \searrow p^{\prime}, r^{\prime}, m^{\prime} \\
& \text { (Stack-in-to-out) } \\
& s \vdash \operatorname{exit\operatorname {Jump}}\left(p, p^{\prime}\right) \\
& m^{\prime}=m\left[\mathrm{SP}_{\mathrm{sec}} \mapsto r(\mathrm{SP})\right] \quad r^{\prime}=r\left[\mathrm{SP} \mapsto m\left(\mathrm{SP}_{\mathrm{ext}}\right)\right] \\
& s \vdash \text { protected } r(\mathrm{SP}) \quad s \vdash \text { unprotected } r^{\prime}(\mathrm{SP}) \\
& p, r, m, s \vdash \text { setStack } \searrow p^{\prime}, r^{\prime}, m^{\prime} \\
& \text { (Stack-no-change-i) } \\
& s \vdash \operatorname{int} \operatorname{Jump}\left(p, p^{\prime}\right) \quad s \vdash \text { protected } r(\mathrm{SP}) \\
& p, r, m, s \vdash \text { setStack } \searrow p^{\prime}, r, m \\
& \text { (Stack-no-change-e) } \\
& s \vdash \operatorname{ext} \operatorname{Jump}\left(p, p^{\prime}\right) \quad s \vdash \text { unprotected } r(\mathrm{SP}) \\
& p, r, m, s \vdash \text { setStack } \searrow p^{\prime}, r, m
\end{aligned}
$$

Figure 4: Stack switch enforcement rules.

addresses $p$ and $p^{\prime}$, the stack switch rules produce a new register file $r^{\prime}$ and a new memory $m^{\prime}$ based on old ones $r$ and $m$. The memory is updated to store the top of the current stack, located in SP, in the address storing the top of the current stack: $\mathrm{SP}_{\text {sec }}$ or $\mathrm{SP}_{\text {ext }}$. When the stack is changed, the register file is updated to initialise SP to the top of the right stack: the address stored at $\mathrm{SP}_{\mathrm{sec}}$ or $\mathrm{SP}_{\text {ext }}$.

The operational semantics is a small step semantics that describes how each instruction of the language transforms an execution state into a new one. Thus, the operational semantics handles programs in the whole memory: both the protected and unprotected partitions.

Definition 1 (EXecution StATE). An execution state, denoted as $\Omega$, is a quintuple $\Omega=(p, r, f, m, s)$, where $p$ is a program counter, $r$ is a register file, $f$ is a flags register, $m$ is a memory and $s$ is a memory descriptor.

Given $\Omega=(p, r, f, m, s)$, let $\lfloor\Omega\rfloor$ be the state $\left(p, r, f, \mathrm{~m}_{\mathrm{sec}}(m, s), s\right)^{p}$ and $\lceil\Omega\rceil$ be the state $\left(p, r, f, \mathrm{~m}_{\text {ext }}(m, s), s\right)$. Relations $\stackrel{i}{\rightarrow} \subseteq$ $\lfloor\Omega\rfloor \times\lfloor\Omega\rfloor$ and $\stackrel{e}{\rightarrow} \subseteq\lceil\Omega\rceil \times\lceil\Omega\rceil$ describe the evaluation of instructions that only affect the protected and unprotected parts of memory respectively. Fig. 5 presents the rules for $\stackrel{i}{\rightarrow}$, rules for $\stackrel{e}{\rightarrow}$ are obtained by replacing an intJump assumption with an extJump one. Let $m(p)=$ inst denote that inst is the word allocated in $m(p)$, where inst $\in \mathcal{I}$. To capture termination, the program counter is set to -1 whenever the halt instruction is encountered. This way, no progress can be made, as $m(-1)$ does not return a valid instruction: the program is in a stuck state.

Definition 2 (StuCK StATE). A state $\Omega=(p, r, f, m, s)$ is stuck, denoted as $\Omega^{\perp}$, when the program counter does not point to a valid instruction: $m(p) \notin \mathcal{I}$.

The operational semantics of the language is a binary relation over states $\rightarrow \subseteq \Omega \times \Omega$ defined by the rules of Fig. 6 . Rules Eval-callback and Eval-returnback ensure that the ad-

$$
\begin{aligned}
& \text { (Eval-protected) (Eval-unprotected) } \\
& \frac{\lfloor\Omega\rfloor \stackrel{i}{\rightarrow}\left\lfloor\Omega^{\prime}\right\rfloor}{\Omega \rightarrow \Omega^{\prime}} \quad \frac{\lceil\Omega\rceil \stackrel{e}{\rightarrow}\left\lceil\Omega^{\prime}\right\rceil}{\Omega \rightarrow \Omega^{\prime}} \\
& \text { (Eval-movs-out) } \\
& m(p)=\left(\operatorname{movs} r_{\mathrm{d}} \mathrm{r}_{\mathrm{s}}\right) \quad s \vdash \operatorname{int} \operatorname{Jump}(p, p+1) \\
& s \vdash \operatorname{writeAllowed}\left(p, r\left(\mathrm{r}_{\mathrm{d}}\right)\right) \quad s \vdash \operatorname{unprotected}\left(r\left(\mathrm{r}_{\mathrm{d}}\right)\right) \\
& m^{\prime}=m\left[r\left(r_{\mathrm{d}}\right) \mapsto r\left(r_{\mathrm{s}}\right)\right] \quad r\left(\mathrm{r}_{\mathrm{s}}\right) \in \mathcal{V} \\
& (p, r, f, m, s) \rightarrow\left(p+1, r, f, m^{\prime}, s\right) \\
& \text { (Eval-callback) } \\
& m(p)=\left(\operatorname{call} \mathrm{r}_{\mathrm{d}}\right) \quad p^{\prime}=m\left(r\left(\mathrm{r}_{\mathrm{d}}\right)\right) \quad s \vdash \operatorname{exitJump}\left(p, p^{\prime}\right) \\
& r^{\prime}=r[\mathrm{SP} \mapsto r(\mathrm{SP})+1] \quad m^{\prime}=m[r(\mathrm{SP}) \mapsto p+1] \\
& p, r^{\prime}, m^{\prime}, s \vdash \text { setStack } \searrow p^{\prime}, r^{\prime \prime}, m^{\prime \prime} \\
& \frac{r^{\prime \prime \prime}=r^{\prime \prime}\left[\mathrm{SP} \mapsto r^{\prime \prime}(\mathrm{SP})+1\right] \quad m^{\prime \prime \prime}=m^{\prime \prime}\left[r^{\prime \prime \prime}(\mathrm{SP}) \mapsto \mathrm{A}_{\mathrm{rb}}\right]}{(p, r, f, m, s) \rightarrow\left(p^{\prime}, r^{\prime \prime \prime}, f, m^{\prime \prime \prime}, s\right)} \\
& \text { (Eval-call) } \\
& m(p)=\left(\operatorname{call} \mathrm{r}_{\mathrm{d}}\right) \quad p^{\prime}=m\left(r\left(\mathrm{r}_{\mathrm{d}}\right)\right) \quad s \vdash \operatorname{entryJump}\left(p, p^{\prime}\right) \\
& p, r, m, s \vdash \operatorname{set} \text { tack } \searrow p^{\prime}, r^{\prime}, m^{\prime} \\
& \frac{r^{\prime \prime}=r^{\prime}\left[\mathrm{SP} \mapsto r^{\prime}(\mathrm{SP})+1\right] \quad m^{\prime \prime}=m^{\prime}\left[r^{\prime \prime}(\mathrm{SP}) \mapsto p+1\right]}{(p, r, f, m, s) \rightarrow\left(p^{\prime}, r^{\prime \prime}, f, m^{\prime \prime}, s\right)} \\
& \text { (Eval-returnback) } \\
& m(p)=(\text { ret }) \quad p^{\prime}=m(r(\mathrm{SP}))=\mathrm{A}_{\mathrm{rb}} \quad s \vdash \operatorname{entryJump}\left(p, p^{\prime}\right) \\
& r^{\prime}=r[\mathrm{SP} \mapsto r(\mathrm{SP})-1] \quad p, r^{\prime}, m, s \vdash \operatorname{setStack} \searrow p^{\prime}, r^{\prime \prime}, m^{\prime} \\
& (p, r, f, m, s) \rightarrow\left(p^{\prime}, r^{\prime \prime}, f, m^{\prime}, s\right) \\
& \text { (Eval-return) } \\
& m(p)=(\text { ret }) \quad p^{\prime}=m(r(\mathrm{SP})) \quad s \vdash \operatorname{exitJump}\left(p, p^{\prime}\right) \\
& \frac{r^{\prime}=r[\mathrm{SP} \mapsto r(\mathrm{SP})-1] \quad p, r^{\prime}, m, s \vdash \text { setStack } \searrow p^{\prime}, r^{\prime \prime}, m^{\prime}}{(p, r, f, m, s) \rightarrow\left(p^{\prime}, r^{\prime \prime}, f, m^{\prime}, s\right)}
\end{aligned}
$$

Figure 6: Operational semantics of whole programs.

dress to be followed after a callback is stored in the secure stack and that the address returnback entry point $A_{r b}$ is pushed on the insecure stack. Thus the unprotected code always jumps to the returnback entry point when returning from a callback. Code located at the returnback entry point must contain a ret instruction in order to correctly resume the execution. Rule Eval-movs-out ensures that values written in unprotected memory are not instructions.

The transitive closure of relation $\rightarrow$ is indicated with $\rightarrow^{*}$. A state $\Omega$ performing $n$ reduction steps is indicated as $\Omega \rightarrow^{n}$ $\Omega^{\prime}$. The evaluation of program $P$ is a sequence of steps that takes the initial state of $P$ to another state.

DeFinition 3 (Initial State). The initial state of a program $(m, s)$, denoted as $\Omega_{0}(m, s)$, is the state $\left(p_{0}, r_{0}, f_{0}, m, s\right)$, where $s=\left(a_{b}, n_{c}, n_{d}, n\right), p_{0}=\left(a_{b}+n_{c}+n_{d}+2\right), r_{0}=[\mathrm{SP} \mapsto$ $\left.m\left(\mathrm{SP}_{\text {ext }}\right) ; r_{\mathrm{i}} \mapsto 0_{\mathrm{i}=0 . .11}\right]$, and $f_{0}=[\mathrm{ZF} \mapsto 0 ; \mathrm{SF} \mapsto 0]$.

The evaluation of $P$ terminates if $\Omega_{0}(P) \rightarrow^{*} \Omega^{\perp}$; the result is stored in $r_{0}$. If the evaluation of program $P$ does not 


$$
\begin{aligned}
& \text { (Eval-movl) } \\
& m(p)=\left(\operatorname{movl} r_{\mathrm{d}} \mathrm{r}_{\mathrm{s}}\right) \\
& s \vdash \operatorname{int} \operatorname{Jump}(p, p+1) \\
& s \vdash \operatorname{readAllowed}\left(p, r\left(\mathrm{r}_{\mathrm{s}}\right)\right) \\
& r^{\prime}=r\left[\mathrm{r}_{\mathrm{d}} \mapsto m\left(r\left(\mathrm{r}_{\mathrm{s}}\right)\right)\right] \\
& (p, r, f, m, s) \stackrel{i}{\rightarrow}\left(p+1, r^{\prime}, f, m, s\right) \\
& \text { (Eval-compare) } \\
& m(p)=\left(\begin{array}{lll}
\operatorname{cmp} r_{1} r_{2}
\end{array}\right) \quad s \vdash \operatorname{int} \operatorname{Jump}(p, p+1) \\
& m(p) \stackrel{(\text { Eval-movs })}{=}\left(\text { movs } r_{\mathrm{d}} \mathbf{r}_{\mathrm{s}}\right) \\
& s \vdash \operatorname{int} \operatorname{Jump}(p, p+1) \\
& s \vdash \operatorname{writeAllowed}\left(p, r\left(\mathrm{r}_{\mathrm{d}}\right)\right) \\
& m^{\prime}=m\left[r\left(r_{\mathrm{d}}\right) \mapsto r\left(\mathrm{r}_{\mathrm{s}}\right)\right] \\
& (p, r, f, m, s) \stackrel{i}{\rightarrow}\left(p+1, r, f, m^{\prime}, s\right) \\
& \left.\mathrm{SF} \mapsto\left(r_{1}<r_{2}\right)\right] \\
& \frac{f^{\prime}=f\left[\mathrm{ZF} \mapsto\left(\mathrm{r}_{1}==\mathrm{r}_{2}\right) ; \quad \mathrm{SF} \mapsto\left(\mathrm{r}_{1}\right.\right.}{(p, r, f, m, s) \stackrel{i}{\rightarrow}\left(p+1, r, f^{\prime}, m, s\right)} \\
& \text { (Eval-sub) } \\
& m(p)=\left(\begin{array}{l}
\text { sub } r_{\mathrm{d}} \mathrm{r}_{\mathrm{s}}
\end{array}\right) \\
& s \vdash \operatorname{int} \operatorname{Jump}(p, p+1) \\
& v=\left(r\left(\mathrm{r}_{\mathrm{d}}\right)-r\left(\mathrm{r}_{\mathrm{s}}\right)\right) \% 2^{\ell} \quad r^{\prime}=r\left[\mathrm{r}_{\mathrm{d}} \mapsto v\right] \\
& f^{\prime}=f[\mathrm{ZF} \mapsto(v==0) ; \\
& \left.\mathrm{SF} \mapsto\left(r\left(\mathrm{r}_{\mathrm{d}}\right)-r\left(\mathrm{r}_{\mathrm{s}}\right)<0\right)\right] \\
& (p, r, f, m, s) \stackrel{i}{\rightarrow}\left(p+1, r^{\prime}, f^{\prime}, m, s\right) \\
& \text { (Eval-je-true) } \\
& m(p)=\left(\begin{array}{ll}
\text { je } r_{\mathrm{i}}
\end{array}\right) \quad f(\mathrm{ZF})==1 \\
& p^{\prime}=r\left(\mathrm{r}_{\mathrm{i}}\right) \quad s \vdash \operatorname{intJump}\left(p, p^{\prime}\right) \\
& (p, r, f, m, s) \stackrel{i}{\rightarrow}\left(p^{\prime}, r, f, m, s\right) \\
& \text { (Eval-jl-false) } \\
& m(p)=\left(\begin{array}{ll}
j 1 & r_{\mathrm{i}}
\end{array}\right) \quad f(\mathrm{SF})==0 \\
& s \vdash \operatorname{int} \operatorname{Jump}(p, p+1) \\
& (p, r, f, m, s) \stackrel{i}{\rightarrow}(p+1, r, f, m, s)
\end{aligned}
$$

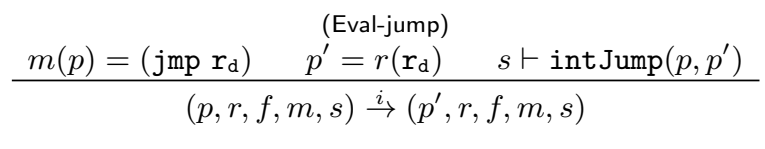

$$
\begin{aligned}
& m(p)=\left(\text { add } \mathrm{r}_{\mathrm{d}} \mathrm{r}_{\mathrm{s}}\right) \quad s \vdash \operatorname{int} \operatorname{Jump}(p, p+1) \\
& m(p)=\left(\text { movi } \mathbf{r}_{\mathrm{d}} \mathbf{i}\right) \\
& s \vdash \operatorname{int} \operatorname{Jump}(p, p+1) \\
& r^{\prime}=r\left[r_{\mathrm{d}} \mapsto i\right] \\
& (p, r, f, m, s) \stackrel{i}{\rightarrow}\left(p+1, r^{\prime}, f, m, s\right) \\
& \text { (Eval-add) } \\
& \frac{v=\left(r\left(r_{\mathrm{d}}\right)+r\left(\mathrm{r}_{\mathrm{s}}\right)\right) \% 2^{\ell} \quad r^{\prime}=r\left[\mathrm{r}_{\mathrm{d}} \mapsto v\right] \quad f^{\prime}=f[\mathrm{ZF} \mapsto(v==0)]}{(p, r, f, m, s) \stackrel{i}{\rightarrow}\left(p+1, r^{\prime}, f^{\prime}, m, s\right)} \\
& \text { (Eval-function-call) } \\
& \begin{aligned}
m(p)= & \left(\operatorname{call} \mathrm{r}_{\mathrm{d}}\right) \quad p^{\prime} \\
s & \vdash \operatorname{intJump}\left(p, p^{\prime}\right)
\end{aligned}=r\left(\mathrm{r}_{\mathrm{d}}\right)
\end{aligned}
$$

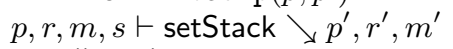

$$
\begin{aligned}
& r^{\prime \prime}=r^{\prime}[\mathrm{SP} \mapsto r(\mathrm{SP})+1] \\
& m^{\prime \prime}=m^{\prime}\left[r^{\prime \prime}(\mathrm{SP}) \mapsto p+1\right] \\
& (p, r, f, m, s) \stackrel{i}{\rightarrow}\left(p^{\prime}, r^{\prime \prime}, f, m^{\prime \prime}, s\right) \\
& \text { (Eval-jl-true) } \\
& m(p)=\left(\begin{array}{ll}
j l & r_{\mathrm{i}}
\end{array}\right) \quad f(\mathrm{SF})==1 \\
& \frac{p^{\prime}=r\left(\mathrm{r}_{\mathrm{i}}\right) \quad s \vdash \operatorname{int} \operatorname{Jump}\left(p, p^{\prime}\right)}{(p, r, f, m, s) \stackrel{i}{\rightarrow}\left(p^{\prime}, r, f, m, s\right)} \\
& \begin{array}{c}
m(p)=\left(\text { je }_{\mathrm{i}}\right) \quad f(\mathrm{ZF})==0 \\
s \vdash \text { int Jump }(p, p+1) \\
\hline(p, r, f, m, s) \stackrel{i}{\rightarrow}(p+1, r, f, m, s) \\
\text { (Eval-halt) }
\end{array} \\
& m(p)=(\text { ret }) \quad p^{\prime}=r(\mathrm{SP}) \\
& s \vdash \operatorname{int} \operatorname{Jump}\left(p, p^{\prime}\right) \\
& r^{\prime}=r[\mathrm{SP} \mapsto r(\mathrm{SP})-1] \\
& \frac{p, r^{\prime}, m, s \vdash \text { setStack } \searrow p^{\prime}, r^{\prime \prime}, m^{\prime}}{(p, r, f, m, s) \stackrel{i}{\rightarrow}\left(p^{\prime}, r^{\prime \prime}, f, m^{\prime}, s\right)} \\
& \text { (Eval-je-false) } \\
& \frac{m(p)=(\text { halt })}{(p, r, f, m, s) \stackrel{i}{\rightarrow}(-1, r, f, m, s)}
\end{aligned}
$$

\begin{tabular}{|c|c|}
\hline 100 & movi $r_{0} 150$ \\
\hline 10 & movs $r_{0} r_{1}$ \\
\hline 10 & movi $r_{0} 0$ \\
\hline 10 & ret \\
\hline
\end{tabular}

\section{Figure 5: Operational semantics of instructions in the protected memory partition.}

terminate, $P$ diverges. A program $P$ diverges, denoted as $P \Uparrow$, if it executes an unbounded number of reduction steps. Formally: $P \Uparrow$ if $\forall n \in \mathbb{N}, \exists \Omega^{\prime} . \Omega_{0}(P) \rightarrow^{n} \Omega^{\prime}$.

\subsection{Contextual Equivalence}

Contextual equivalence relates two programs that cannot be distinguished by any third program interacting with them [15]. This notion relies on the concept of contexts, which is introduced before presenting the equivalence itself.

Since we consider programs $P$ that are placed in protected memory and interact with arbitrary unprotected code, contexts model that unprotected code. Thus for any descriptor $s$, contexts $\mathbb{M}$ are partial memories with a hole: $\mathbb{M}=m[\cdot]$, where all addresses of $\mathbb{M}$ are unprotected. Formally, given $s$, $\forall a \in \operatorname{dom}(\mathbb{M}), s \vdash$ unprotected $(a)$. The hole models the possibility to combine a program $P$ with the memory $\mathbb{M}$ iff they are compatible, denoted as $P \frown \mathbb{M}$, thus if the memories of $P$ and $\mathbb{M}$ have disjoint domains. Let $\operatorname{dom}(\mathbb{M})=\operatorname{dom}(m)$ if $\mathbb{M}=m[\cdot]$; formally, $P \frown \mathbb{M}$ if $P=\left(m^{\prime}, s\right)$ and $\operatorname{dom}\left(m^{\prime}\right) \cap$ $\operatorname{dom}(\mathbb{M})=\emptyset$. If $P$ and $\mathbb{M}$ are compatible, the hole of $\mathbb{M}$ can be filled with $P$ in order to model interaction between $P$ and $\mathbb{M}$. Formally, if $P \frown \mathbb{M}$ then $\mathbb{M}\left[\left(m^{\prime}, s\right)\right]=\left(m^{\prime}+m, s\right)$.

Programs $P_{1}$ and $P_{2}$ are contextually equivalent, denoted as $P_{1} \simeq P_{2}$, when, for all contexts they interact with, $P_{1}$ diverges if and only if $P_{2}$ also diverges.

Definition 4 (Contextual Equivalence). $P_{1} \simeq P_{2}$ if $\forall \mathbb{M}$. $P_{1} \frown \mathbb{M} \wedge \mathbb{M}\left[P_{1}\right] \Uparrow \Longleftrightarrow P_{2} \frown \mathbb{M} \wedge \mathbb{M}\left[P_{2}\right] \Uparrow$.

An implication of this definition is that for $P_{1}$ and $P_{2}$ to be contextually equivalent they must have the same memory descriptor. For the sake of simplicity we will always assume the compatibility of a program and the context it is plugged in, shortening the above definition to: $P_{1} \simeq P_{2}$ if $\forall \mathbb{M} . \quad \mathbb{M}\left[P_{1}\right] \Uparrow \Longleftrightarrow \mathbb{M}\left[P_{2}\right] \Uparrow$.
EXAmple 2 (CONTEXTUAlly EQUivalent PROGRAMS). The following $P_{L}$ and $P_{R}$ write the values of $r_{1}$ and $r_{2}$ respectively at the protected address 150 (line 2) and then return 0 (line 3). Recall that the protected memory partition spans from address 100 to 200, with one entry point at address 100.

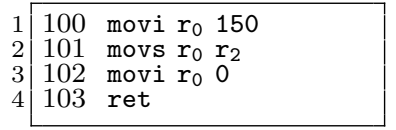

The only difference between $P_{L}$ and $P_{R}$ is in the value stored at address 150. However, an unprotected program cannot read that value. Since that value does not affect the computation of $P_{L}$ or $P_{R}$ or the unprotected code, $P_{L}$ and $P_{R}$ are contextually equivalent.

Having defined the assembly language and its operational semantics, the paper introduces the two different trace semantics. Trace equivalence is also introduced, it will be proven the same as contextual equivalence in Section 5.

\section{TRACE SEMANTICS}

This section gives two different trace semantics for protected programs. The differences stem from the different ways to achieve full abstraction pointed out by Curien [3]. The first, $\operatorname{Tr}^{\mathrm{L}}$, possesses more expressive labels, while the second, $\operatorname{Tr}^{\mathrm{S}}$, relies on changes to the semantics of programs. Both are proven to be fully abstract w.r.t. the appropriate operational semantics in Section 5. Finally, this section defines when two programs are trace equivalent.

As for the operational semantics, a notion of execution states is required for the trace semantics as well. Execution states for $\operatorname{Tr}^{\mathrm{L}}$ and $\operatorname{Tr}^{\mathrm{S}}$, denoted as $\Theta$, are the same as $\Omega$ except that $\Theta$ do not deal with the whole memory but just with 
its protected partition. So, the memory $m$ of $(p, r, f, m, s)$ spans only the protected memory partition indicated by $s$. Additionally, $\Theta$ can be (unknown, $m, s$ ), an unknown state modelling code executing in unprotected memory.

DEFINITION 5 (INITIAL STATE FOR TRACES). The initial state for traces of a program $(m, s)$, denoted as $\Theta_{0}(m, s)$, is the state (unknown, $m, s$ ).

\section{1 $\operatorname{Tr}^{\mathrm{L}}$ : Expressive Labels}

Below are the labels exhibited by the $T r^{\mathrm{L}}$ traces semantics.

$$
\begin{array}{ll}
\Lambda::=\alpha \mid \tau_{i} & \alpha::=\sqrt{ }|\gamma ?| \delta ! \\
\gamma::=(r ; f) \operatorname{call} a \mid(r ; f) \text { ret } a & \delta::=\operatorname{write}(a, v) . \delta \mid \gamma
\end{array}
$$

A label $\Lambda$ can be either an observable action $\alpha$ or a nonobservable action $\tau_{i}$. Action $\tau_{i}$ indicates the unobservable action occurred in protected memory. Observable actions include a tick $\sqrt{ }$ indicating that the evaluation has terminated. Additionally, observable actions are function calls or returns to a certain address $a$, combined with the registers $r$ and flags $f$. Registers and flags are in the labels as they convey information on the behaviour of programs. Observable, !-decorated actions can be prefixed by a number of writeouts of a value $v$ to address $a$. They are only !-decorated since the protection mechanism forbids writes from unprotected to protected memory, as well as read-outs.

Fig. 7 presents the rules defining the relation $\Theta \stackrel{\bar{\alpha}}{\Longrightarrow} \Theta^{\prime}$, which describe when a state $\Theta$ generates trace $\bar{\alpha}$ and results in state $\Theta^{\prime}$. The $\operatorname{Tr}^{\mathrm{L}}$ traces of a program $P$ is defined as follows: $\operatorname{Tr}^{\mathrm{L}}(P)=\left\{\bar{\alpha} \mid \exists \Theta^{\prime} . \Theta_{0}(P) \stackrel{\bar{\alpha}}{\Longrightarrow} \Theta^{\prime}\right\}$.

Example 3 below can now explain why protected code cannot write instructions in unprotected memory.

EXAmple 3 (Write-OUts ARE not instructions). In the following example, protected programs can write instructions in unprotected memory. Recall that the protected memory partition spans from address 100 to 200, with one entry point at address 100.

The following $P_{L}$ and $P_{R}$ set $r_{0}$ to 20 and 10 respectively (line 1), then write the instruction jmp $r_{0}$ at address 20 and 10 respectively (line 2). Finally, they jump to the instruction they just wrote (line 3).

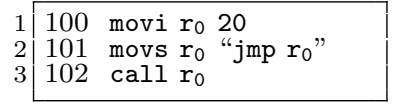

\begin{tabular}{llll|}
\hline 1 & 100 & movi $r_{0}$ & 10 \\
2 & 101 & movs $r_{0}$ & jmp $r_{0} "$ \\
3 & 102 & call $r_{0}$ & \\
& & &
\end{tabular}

When $\mathrm{r}_{0}$ is set to 20 (resp. 10), the instruction jmp $\mathrm{r}_{0}$ written at address 20 (resp. 10) will diverge. Thus, $P_{L}$ and $P_{R}$ are contextually equivalent, since no context can differentiate between them. However, $P_{L}$ and $P_{R}$ are trace inequivalent, since the following is a trace of $P_{L}$ and not of $P_{R}:(\cdots)$ call 100? · write $\left(20\right.$, "jmp $\left.r_{0}{ }^{\prime \prime}\right) .(\cdot)$ call 20 !

Since we are interested in programs that do not extend the functionality of code in unprotected memory (and since these programs are the majority of assembly programs), protected code cannot write instructions in unprotected memory. Thus the programs of this example are not valid.

\section{2 $T_{r^{s}}$ : Changes to the Semantics}

The $T r^{\mathrm{S}}$ semantics presents different labels from $\operatorname{Tr}^{\mathrm{L}}$, which are driven by changes to the operational semantics of protected programs. These changes are formalised in Fig. 8; smaller changes to other rules are omitted. When the pro-

$$
\begin{aligned}
& \text { (Trace-internal) } \\
& \frac{(p, r, f, m, s) \stackrel{i}{\rightarrow}\left(p^{\prime}, r^{\prime}, f^{\prime}, m^{\prime}, s\right) \quad s \vdash \operatorname{int} \operatorname{Jump}\left(p, p^{\prime}\right)}{(p, r, f, m, s) \stackrel{\tau_{i}}{\longrightarrow}\left(p^{\prime}, r^{\prime}, f^{\prime}, m^{\prime}, s\right)} \\
& \text { (Trace-internal-tick) } \\
& (p, r, f, m, s) \stackrel{i}{\rightarrow}\left(p^{\prime}, r^{\prime}, f^{\prime}, m^{\prime}, s\right) \\
& \frac{s \vdash \operatorname{protected}(p) \quad\left(p^{\prime}, r^{\prime}, f^{\prime}, m^{\prime}, s\right)^{\perp}}{(p, r, f, m, s) \stackrel{\vee}{\longrightarrow}\left(p^{\prime}, r^{\prime}, f^{\prime}, m^{\prime}, s\right)} \\
& \text { (Trace-writeout-accumulate) } \\
& s \vdash \operatorname{int} \operatorname{Jump}(p, p+1) \quad m(p)=\left(\operatorname{movs} \mathbf{r}_{\mathrm{d}} \mathbf{r}_{\mathbf{s}}\right) \\
& s \vdash \operatorname{unprotected}\left(r\left(\mathrm{r}_{\mathrm{d}}\right)\right) \quad r\left(\mathrm{r}_{\mathrm{s}}\right) \in \mathcal{V} \\
& \frac{(p+1, r, f, m, s) \stackrel{\delta ! ~}{\Longrightarrow}\left(p^{\prime}, r^{\prime}, f^{\prime}, m^{\prime}, s\right)}{(p, r, f, m, s) \stackrel{\text { write }\left(r\left(\mathrm{r}_{\mathrm{d}}\right), r\left(\mathrm{r}_{\mathrm{s}}\right)\right) . \delta !}{\longrightarrow}\left(p^{\prime}, r^{\prime}, f^{\prime}, m^{\prime}, s\right)} \\
& \text { (Trace-writeout-discard) } \\
& s \vdash \operatorname{int} \operatorname{Jump}(p, p+1) \quad m(p)=\left(\text { movs } r_{\mathrm{d}} \boldsymbol{r}_{\mathrm{s}}\right) \\
& s \vdash \text { unprotected }\left(r\left(r_{\mathrm{d}}\right)\right) \\
& \frac{(p+1, r, f, m, s) \stackrel{\sqrt{ }}{\Longrightarrow}\left(p^{\prime}, r^{\prime}, f^{\prime}, m^{\prime}, s\right)}{(p, r, f, m, s) \stackrel{\sqrt{ }}{\longrightarrow}\left(p^{\prime}, r^{\prime}, f^{\prime}, m^{\prime}, s\right)} \\
& \text { (Trace-call) } \\
& s \vdash \text { entryPoint }(p) \\
& \text { (unknown, } m, s) \stackrel{(r ; f) \text { call } p \text { ? }}{\longrightarrow}(p, r, f, m, s) \\
& \text { (Trace-returnback) } \\
& s \vdash \text { returnEntryPoint }(p) \\
& \text { (unknown, } m, s) \stackrel{(r ; f) \text { ret } p ?}{\longrightarrow}(p, r, f, m, s) \\
& \text { (Trace-callback) } \\
& s \vdash \operatorname{exitJump}\left(p, p^{\prime}\right) \quad m(p)=\left(\operatorname{call} p^{\prime}\right) \\
& \frac{r^{\prime}=r[\mathrm{SP} \mapsto r(\mathrm{SP})+1] \quad m^{\prime}=m[r(\mathrm{SP}) \mapsto p+1]}{\left.(p, r, f, m, s) \stackrel{(r ; f) \text { call } p^{\prime} !}{\longrightarrow} \text { (unknown, } m^{\prime}, s\right)} \\
& \text { (Trace-return) } \\
& p^{\prime}=m(\mathrm{SP}) \quad s \vdash \operatorname{exitJump}\left(p, p^{\prime}\right) \quad m(p)=(\text { ret }) \\
& \left.(p, r, f, m, s) \stackrel{(r ; f) \text { ret } p^{\prime} !}{\longrightarrow} \text { (unknown, } m, s\right)
\end{aligned}
$$

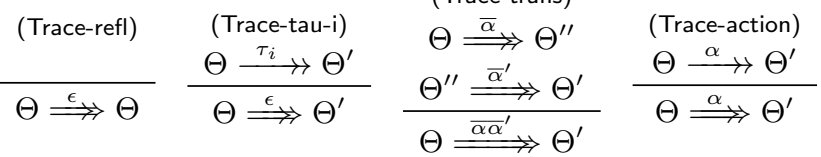

Figure 7: Rules of the $\operatorname{Tr}^{L}$ trace semantics.

gram counter jumps between the protected and the unprotected memory partitions, or vice-versa, flags are set to 0; in case of a return, all registers but $R_{0}$ are also set to 0 . Additionally, write-outs are prohibited.

Following are the labels of $\operatorname{Tr}^{\mathrm{S}}$; we drop the greek letter notation and use latin letters in order to immediately differentiate between $T r^{\mathrm{L}}$ and $T r^{\mathrm{S}}$. Flags do not appear in traces because they are always set to 0 , as are all registers but $R_{0}$ in case of a return. Write-outs are prohibited, so there are no labels that capture them.

$$
L::=a\left|\tau_{i} \quad a::=\sqrt{ }\right| g ? \mid g ! \quad g::=\text { call } p(r) \mid \text { ret } p r\left(r_{0}\right)
$$

Rules defining the relation $\Theta \stackrel{\bar{a}}{\Longrightarrow} \Theta^{\prime}$ for $\operatorname{Tr}^{\mathrm{S}}$ are omitted. They are a subset of the ones defined in Fig. 7, with a slight modification to the syntax of generated labels. The $\operatorname{Tr}^{\mathrm{S}}$ traces of a program $P$ is defined as follows: $\operatorname{Tr}^{\mathrm{S}}(P)=$ $\left\{\bar{a} \mid \exists \Theta . \Theta_{0}(P) \stackrel{\bar{a}}{\Longrightarrow} \Theta\right\}$.

\subsection{Trace Equivalence}

The notion of trace equivalence is presented generically for both trace semantics under consideration. Use $\operatorname{Tr}(P)$ to 


$$
\begin{aligned}
& \text { (Aux-write-1') } \\
& s \vdash \operatorname{unprotected}(p) \quad s \vdash \operatorname{unprotected}(a) \\
& s \vdash \text { writeAllowed }(p, a) \\
& \text { (Stack-out-to-in') } \\
& s \vdash \operatorname{entry\operatorname {Jump}}\left(p, p^{\prime}\right) \quad m^{\prime}=m\left[\mathrm{SP}_{\text {ext }} \mapsto r(\mathrm{SP})\right] \\
& r^{\prime}=r\left[\mathrm{SP} \mapsto m\left(\mathrm{SP}_{\mathrm{sec}}\right)\right] \quad f^{\prime}=[\mathrm{ZF} \mapsto 0 ; \mathrm{SF} \mapsto 0] \\
& s \vdash \text { unprotected } r(\mathrm{SP}) \quad s \vdash \text { protected } r^{\prime}(\mathrm{SP}) \\
& p, r, f, m, s \vdash \text { setStack } \searrow p^{\prime}, r^{\prime}, f^{\prime}, m^{\prime} \\
& \text { (Stack-in-to-out') } \\
& s \vdash \operatorname{exitJump}\left(p, p^{\prime}\right) \quad m^{\prime}=m\left[\mathrm{SP}_{\mathrm{sec}} \mapsto r(\mathrm{SP})\right] \\
& r^{\prime}=r\left[\mathrm{SP} \mapsto m\left(\mathrm{SP}_{\mathrm{ext}}\right)\right] \quad f^{\prime}=[\mathrm{ZF} \mapsto 0 ; \mathrm{SF} \mapsto 0] \\
& s \vdash \text { protected } r(\mathrm{SP}) \quad s \vdash \text { unprotected } r^{\prime}(\mathrm{SP}) \\
& p, r, f, m, s \vdash \text { setStack } \searrow p^{\prime}, r^{\prime}, f^{\prime}, m^{\prime} \\
& \text { (Eval-return') } \\
& m(p)=(\text { ret }) \quad p^{\prime}=m(r(\mathrm{SP})) \quad s \vdash \operatorname{exitJump}\left(p, p^{\prime}\right) \\
& r^{\prime}=r\left[\mathrm{SP} \mapsto r(\mathrm{SP})-1 ; \mathrm{R}_{\mathrm{i}} \mapsto 0_{\mathrm{i}=0 . .11}\right] \\
& p, r^{\prime}, f, m, s \vdash \text { setStack } \searrow p^{\prime}, r^{\prime \prime}, f^{\prime}, m^{\prime} \\
& (p, r, f, m, s) \rightarrow\left(p^{\prime}, r^{\prime \prime}, f^{\prime}, m^{\prime}, s\right)
\end{aligned}
$$

Figure 8: Changes to auxiliary functions and to the operational semantics for $\operatorname{Tr}^{S}$.

indicate the traces of a program $P$, be it expressed through $\operatorname{Tr}^{\mathrm{L}}$ or $\mathrm{Tr}^{\mathrm{S}}$.

Two programs $P_{1}$ and $P_{2}$ are trace-equivalent, denoted as $P_{1} \simeq_{\mathrm{T}} P_{2}$, if their traces are the same and they have the same memory descriptor.

Definition 6 (Trace equivalence). $P_{1} \simeq_{\mathrm{T}} P_{2}$ if $\operatorname{Tr}\left(P_{1}\right)=\operatorname{Tr}\left(P_{2}\right)$ and $P_{1}=\left(m_{1}, s\right)$ and $P_{2}=\left(m_{2}, s\right)$.

Following are two examples of trace equivalent and inequivalent programs. For the sake of simplicity, we use the $\operatorname{Tr}^{\mathrm{L}}$ semantics and indicate arbitrary values for registers and flags with notation $(r, f)$ and an unprotected address with $p$.

Example 4 (Traces of Previous examples). Code in Example 1 is not trace equivalent; the following trace is generated by $P_{L}$ but not by $P_{R}:(r ; f)$ call $100 ? \cdot(. ., 41 ; f)$ ret $p$ ! $\sqrt{ }$. The code of Example 2 is trace equivalent since the trace semantics of both $P_{L}$ and $P_{R}$ is a set whose sequences are concatenations of the following trace, each element of the sequence being parametrised on $r$ and $f:(r ; f)$ call 100? . $\left(r\left[\mathrm{R}_{0} \mapsto 0\right] ; f\right)$ ret $p$ !

\section{FULL ABSTRACTION THEOREM}

This section presents the general proof strategy through which both $\operatorname{Tr}^{\mathrm{L}}$ and $\operatorname{Tr}^{\mathrm{S}}$ are proven to be fully abstract w.r.t. the corresponding operational semantics and discusses the benefits of full abstraction of the trace semantics.

A fully abstract trace semantics is both sound and complete with respect to the operational semantics. Soundness states that the trace semantics captures all details of the operational semantics. Thus, for all contexts, two trace equivalent programs cannot be told apart. The universal quantification over contexts makes this more difficult to prove. Completeness states that abstractions of the trace semantics are preserved in the operational semantics. This is achieved by proving the contrapositive of the completeness statement. For this, the proof consists of devising an algorithm that constructs a program, a "witness", that tells two trace inequivalent programs apart $[2,6,14]$. Since the language is low-level and untyped, this is not particularly complicated.
Full abstraction of trace semantics is formally stated as: $P_{1} \simeq_{\mathrm{T}} P_{2} \Longleftrightarrow P_{1} \simeq P_{2}$; its proof is split in two cases, one for each direction of the co-implication.

Theorem 1 (Soundness). $P_{1} \simeq_{\mathrm{T}} P_{2} \Rightarrow P_{1} \simeq P_{2}$.

Call the interface of a state its registers, flags and unprotected memory. Two states $\Omega_{1}$ and $\Omega_{2}$ have the same interface, denoted as $\Omega_{1} \doteq \Omega_{2}$, if they have the same registers, flags and unprotected memory. Formally, $\Omega_{1} \stackrel{\circ}{=} \Omega_{2}$ if $\Omega_{1}=$ $\left(p_{1}, r, f, m_{1}, s_{1}\right)$ and $\Omega_{2}=\left(p_{2}, r, f, m_{2}, s_{2}\right)$ and $\mathrm{m}_{\text {ext }}\left(m_{1}, s_{1}\right)=$ $\mathrm{m}_{\text {ext }}\left(m_{2}, s_{2}\right)$. Given $\Omega=(p, r, f, m, s)$, redefine $\lfloor\Omega\rfloor$ to be state $\Theta=\left(p, r, f, \mathrm{~m}_{\mathrm{sec}}(m, s), s\right)$ if $s \vdash \operatorname{protected}(p)$ and (unknown, $m, s$ ) otherwise.

The proof of Theorem 1 states that an unprotected program interacting with $P_{1}$ cannot distinguish it from $P_{2}$. This is because both programs offer the same interface to the unprotected program. So this proof depends on an interfacepreservation lemma (Lemma 1) which must be proven for each trace semantics since it depends on the labels of each trace semantics. Lemma 1 enunciates that two states with the same interface still have the same interface after they perform the same observable action. Thus unprotected programs do not see differences, in terms of flags, registers and unprotected memory, between $P_{1}$ and $P_{2}$.

$$
\begin{aligned}
& \text { LEMma } 1 \text { (INTERFACE PRESERVATION). If } \Theta_{1} \stackrel{\bar{\alpha}}{\Longrightarrow} \\
& \stackrel{\alpha}{\longrightarrow} \Theta_{1}^{\prime} \text { and } \Theta_{1}=\left\lfloor\Omega_{1}\right\rfloor \text { and } \Omega_{1} \rightarrow^{*} \Omega_{1}^{\prime} \text { and } \Theta_{1}^{\prime}=\left\lfloor\Omega_{1}^{\prime}\right\rfloor
\end{aligned}
$$
and $\Theta_{2} \stackrel{\bar{\alpha}}{\Longrightarrow} \stackrel{\alpha}{\longrightarrow} \Theta_{2}^{\prime}$ and $\Theta_{2}=\left\lfloor\Omega_{2}\right\rfloor$ and $\Omega_{2} \rightarrow^{*} \Omega_{2}^{\prime}$ and $\Theta_{2}^{\prime}=\left\lfloor\Omega_{2}^{\prime}\right\rfloor$ and $\Omega_{1} \stackrel{\circ}{=} \Omega_{2}$ then $\Omega_{1}^{\prime} \stackrel{\circ}{=} \Omega_{2}^{\prime}$.

Theorem 2 (Completeness). $P_{1} \simeq P_{2} \Rightarrow P_{1} \simeq_{\mathrm{T}} P_{2}$.

Completeness is equivalently stated as: $P_{1} \nsucc_{\mathrm{T}} P_{2} \Rightarrow P_{1} \nsucc P_{2}$. This is proven by devising an algorithm that takes as input two different traces $\overline{\alpha_{1}}$ and $\overline{\alpha_{2}}$ and the two programs $P_{1}$ and $P_{2}$ generating them and outputs a program $P$ that interacts with $P_{1}$ and $P_{2}$ and is able to differentiate between them. The two different traces are generated as follows. Since $P_{1} \neq_{\mathrm{T}} P_{2}$, we have that $\operatorname{Tr}\left(P_{1}\right) \neq \operatorname{Tr}\left(P_{2}\right)$, thus there exists a trace $\bar{\alpha}$ that belongs to either only $\operatorname{Tr}\left(P_{1}\right)$ or only $\operatorname{Tr}\left(P_{2}\right)$. Assume wlog that $\bar{\alpha} \in \operatorname{Tr}\left(P_{1}\right)$. The trace $\bar{\alpha}$ can be split in two parts $\overline{\alpha_{s}}$ and $\overline{\alpha_{d}}$ such that $\bar{\alpha}=\overline{\alpha_{s} \alpha_{d}}$, and so that there exists a trace $\overline{\alpha^{\prime}} \in \operatorname{Tr}\left(P_{2}\right)$ that can be split in two parts $\overline{\alpha_{s}}$ and $\overline{\alpha_{d}^{\prime}}$ such that $\overline{\alpha^{\prime}}=\overline{\alpha_{s}} \overline{\alpha_{d}^{\prime}}$ and $\overline{\alpha_{d}} \neq \overline{\alpha_{d}^{\prime}}$. Trace $\overline{\alpha^{\prime}}$ always exists, it could be an empty trace, it could be composed by an empty $\overline{\alpha_{s}}$ and, possibly, by an empty $\overline{\alpha_{d}^{\prime}}$. The traces input for the algorithm are $\overline{\alpha_{1}}=\overline{\alpha_{s} \alpha_{d}}$ and $\overline{\alpha_{2}}=\overline{\alpha_{s}} \overline{\alpha_{d}^{\prime}}$.

The interested reader is referred to the companion technical report for proofs [13]. This general proof strategy is presented for both Theorem 1 and Theorem 2. The generalised approach is tailored to each semantics only in the relatively simple proof of Lemma 1 . Since Theorem 1 and 2 hold for both $\operatorname{Tr}^{\mathrm{L}}$ and $\operatorname{Tr}^{\mathrm{S}}$, both semantics are fully abstract w.r.t. the corresponding operational semantics.

\subsection{Benefits of the Trace Semantics}

Let us now highlight two of the benefits of fully abstract trace semantics.

Without the trace semantics, the capabilities of an attacker towards protected code are expressed as sequences of instructions, which can be difficult to reason about. With the trace semantics, the capabilities of that attacker are captured via the simple notion of traces. The full abstraction property ensures that traces express precisely all the capabilities of an attacker, without leaving any out. 
The second benefit is in the field of secure compilation. Given two programs $C_{1}$ and $C_{2}$ in a language and indicate their compilation to an assembly language with $C_{1}^{\downarrow}$ and $C_{2}^{\downarrow}$ respectively. Proving the compilation scheme secure is formally stated as $C_{1} \simeq C_{2} \Longleftrightarrow C_{1}^{\downarrow} \simeq C_{2}^{\downarrow}$ [1]. The more complex direction of this proof is $C_{1} \simeq C_{2} \Rightarrow C_{1}^{\downarrow} \simeq C_{2}^{\downarrow}$, but it can be simplified by adopting a fully abstract trace semantics for the assembly language. That proof is simpler in this way than by adopting the notion of contextual equivalence and performing induction on all possible low-level contexts. The complex direction becomes $C_{1} \simeq C_{2} \Rightarrow C_{1}^{\downarrow} \simeq_{\mathrm{T}} C_{2}^{\downarrow}$, whose contrapositive is $C_{1}^{\downarrow} \neq_{\mathrm{T}} C_{2}^{\downarrow} \Rightarrow C_{1} \neq C_{2}$. This can be proven similarly to Theorem 2, as Agten et al. [2] and Patrignani et al. [14] did. Both implicitly use the second trace semantics where the compiler adds code that enforces the changes to the operational semantics.

\section{RELATED WORK}

Full abstraction has been largely studied as a way to state the correctness of a denotational semantics with respect to an operational one [15]. This technique has been adopted for different programming languages paradigms, such as the $\lambda$-calculus [10] and the $\pi$-calculus [5].

Fine grained, program counter-based memory access control mechanisms have been implemented in several software [8, $17,18,19]$ and hardware forms $[4,12]$ and recently in the Intel SGX processor [9]. From the theoretical point of view, assembly languages extended with these protection mechanisms have been recently studied as target languages for secure compilation schemes [2, 14]. The language and trace semantics of this paper are inspired by those works. Besides elaborating on details of their formalisation, this work provides the first proof of full abstraction of the trace semantics.

A different research area studies logics for assembly languages: Hoare logics [16] or separation logics [7]. Jensen et al. [7] present a summary of the most recent advances in the latter. That research area focusses on providing reasoning facilities for assembly code, while this paper focusses on reasoning on the security of assembly code.

A different protection mechanism that could be employed at the assembly level is a typed assembly language [11]. To the best of the authors' knowledge, no fully abstract trace semantics has been provided for such languages.

\section{CONCLUSION AND FUTURE WORK}

This paper studied the characterisation of the behaviour of isolated programs. To this extent, it formalised an assembly language extended with a fine grained, program counterbased memory access control mechanism. Then, it provided two different trace semantics for that language and a general proof strategy where both semantics are proven to be fully abstract. These semantics model the capabilities of attackers that inject malicious assembly code and they simplify proving secure compilation to the assembly language.

Providing a fully abstract trace semantics for a machine with multiple instances of an isolation mechanism or with multiple cores seem natural extensions to this work. The latter seems crucial in order to provide a secure compiler for concurrent programs to machines using the protection mechanism presented.

Acknowledgements. The authors would like to thank Tarmo Uustalu and the reviewers for useful feedback on an earlier draft.

\section{REFERENCES}

[1] M. Abadi. Protection in programming-language translations. In Secure Internet programming, pages 19-34. Springer-Verlag, 1999.

[2] P. Agten, R. Strackx, B. Jacobs, and F. Piessens. Secure compilation to modern processors. In CSF '12, pages 171 - 185. IEEE, 2012.

[3] P.-L. Curien. Definability and full abstraction. Electr. Notes Theor. Comput. Sci., 172:301-310, 2007.

[4] K. Eldefrawy, A. Francillon, D. Perito, and G. Tsudik. SMART: Secure and Minimal Architecture for (Establishing a Dynamic) Root of Trust. In NDSS'12.

[5] A. Jeffrey and J. Rathke. Full abstraction for polymorphic pi-calculus. In FOSSACS'05, pages 266-281. Springer-Verlag, 2005.

[6] A. Jeffrey and J. Rathke. A fully abstract may testing semantics for concurrent objects. Theor. Comput. Sci., 338(1-3):17-63, 2005.

[7] J. B. Jensen, N. Benton, and A. Kennedy. High-level separation logic for low-level code. SIGPLAN Not., 48(1):301-314, Jan. 2013.

[8] J. M. McCune, B. J. Parno, A. Perrig, M. K. Reiter, and H. Isozaki. Flicker: an execution infrastructure for TCB minimization. SIGOPS Oper. Syst. Rev., 42(4):315-328, 2008.

[9] F. McKeen et al. Innovative instructions and software model for isolated execution. In $H A S P$ '13, pages 10:1-10:1, New York, NY, USA, 2013. ACM.

[10] R. Milner. Fully abstract models of typed lambda-calculi. Theor. Comput. Sci., 4(1):1-22, 1977.

[11] G. Morrisett, D. Walker, K. Crary, and N. Glew. From system $\mathrm{F}$ to typed assembly language. ACM Trans. Program. Lang. Syst., 21(3):527-568, May 1999.

[12] J. Noorman et al. Sancus: Low-cost trustworthy extensible networked devices with a zero-software Trusted Computing Base. In Proceedings of the 22nd USENIX conference on Security symposium, 2013.

[13] M. Patrignani and D. Clarke. Fully Abstract Trace Semantics of Low-level Protection Mechanisms Extended Version. CW Reports CW 651, Dept. of Computer Science, K.U.Leuven, November 2012.

[14] M. Patrignani, D. Clarke, and F. Piessens. Secure Compilation of Object-Oriented Components to Protected Module Architectures. In (APLAS'13), volume 8301 of $L N C S$, pages 176-191, 2013.

[15] G. Plotkin. LCF considered as a programming language. Theor. Comput. Science, 5:223-255, 1977.

[16] A. Saabas and T. Uustalu. A compositional natural semantics and Hoare logic for low-level languages. Electr. Notes Theor. Comput. Sci., 156:151-168, 2006.

[17] L. Singaravelu, C. Pu, H. Härtig, and C. Helmuth. Reducing TCB complexity for security-sensitive applications: three case studies. SIGOPS Oper. Syst. Rev., 40(4):161-174, 2006.

[18] R. Strackx and F. Piessens. Fides: Selectively hardening software application components against kernel-level or process-level malware. In CCS 2012, pages 2-13. ACM Press, October 2012.

[19] R. Strackx, F. Piessens, and B. Preneel. Efficient isolation of trusted subsystems in embedded systems. In SecureComm, pages 344-361, 2010. 